\title{
Los factores personales como variables determinantes en la formación de la imagen del destino. Especial referencia a las motivaciones turísticas
}

\author{
María José Andrade Suárez* \\ Universidade da Coruña
}

\section{Resumen}

Aunque la mayoría de los recursos turísticos es de carácter objetivo, la imagen percibida de ellos puede ser diversa y heterogénea debido a la naturaleza subjetiva de ésta. El objetivo del presente trabajo consiste en identificar la naturaleza multidimensional de la imagen turística y examinar la influencia de los factores personales del turista (motivaciones, valores culturales y características sociodemográficas) sobre la percepción del destino. Se presta especial atención a los motivos del viajero, entendidos como las fuerzas sociopsicológicas que predisponen a un individuo a optar y participar en una actividad turística. El estudio empírico aplicado al turismo rural en Galicia y realizado por medio de 391 cuestionarios demuestra que existe una relación moderada entre las motivaciones turísticas del viajero y la formación de la imagen afectiva del destino. Asimismo, cuanto más estrecho es el vínculo entre las motivaciones del turista y los atributos emocionales del destino, más positiva es la imagen afectiva de éste.

\section{PAlabras Clave}

Características sociodemográficas, factores personales, formación de la imagen turística, motivaciones, turismo rural, valores culturales. 


\title{
Personal factors as determining variables in forming the image of a tourist destination
}

\author{
María José Andrade Suárez \\ Universidade da Coruña
}

\begin{abstract}
Although most tourist attractions have their objective reality, their image is perceived differently by heterogeneous subjects. The objective of this article is to identify the multidimensional nature of the image tourists have of a place and examine the influence of their personal characteristics (motivations, cultural values and demographic description) on the perception of their destination. I pay special attention to the traveler's motivations, considering them as sociopsychological forces predisposing the traveler to choose to become a tourist. The empirical study was done in rural Galicia where I applied 391 questionnaires. The responses show a moderate relationship between the tourist's motivations and the formation of an affective image of a destination. It is also true that the closer the relationship between the tourist's motivations and the emotional attributes of a destination, the more positive the image of that destination is.
\end{abstract}

\section{KEY WORDS}

Sociodemographic characteristics, personal factors, tourist image formation, motivations, rural tourism, cultural values. 


\section{Introducción}

El estudio de la imagen turística responde a dos aspectos de especial trascendencia; por un lado, el interés de los académicos por explicar científicamente la naturaleza y el proceso de formación de este concepto, así como su influencia en el comportamiento de los turistas, y por otro, la necesidad que manifiestan los promotores de posicionar y diferenciar los destinos en la mente de sus públicos objetivo del modo más eficiente y eficaz posible.

Entre todas las definiciones de la imagen puede destacarse por su amplitud la de Baloglu y McCleary (1999b: 330), quienes la consideran "representación mental de las creencias, los sentimientos y la impresión global del individuo sobre un destino turístico". Esta imagen es generalmente formada por dos fuerzas primordiales: los factores estímulo y los factores personales (Baloglu y McCleary, 1999a). Los primeros se refieren a la cantidad y naturaleza diversa de fuentes de la información a las cuales los individuos están expuestos, incluyendo la información del destino adquirida como consecuencia de haberlo visitado; entre los segundos se encuentran las motivaciones, las características sociodemográficas y la procedencia geográficocultural de los turistas (Beerli Palacio y Martín Santana, 2004a y b).

Se tomará este modelo teórico para realizar una aproximación al efecto moderador de los factores internos del turista sobre la percepción de los destinos, ya que, como argumentan Um y Crompton (1990), las creencias sobre los atributos turísticos se forman al estar expuestos los individuos a estímulos externos, pero la naturaleza de estas creencias varía de acuerdo con sus factores internos. De este modo, cada persona construye su propia y única representación mental del destino turístico que da lugar a una imagen percibida propia y personal (Ashworth y Voogd, 1990; Gartner, 1993; Bramwell y Rawding, 1996).

En este sentido, diversos trabajos atestiguan que la imagen turística se muestra estrechamente afectada por los motivos de los individuos para realizar el viaje (Mayo y Jarvis, 1981; Stabler, 1990; Um y Crompton, 1990; Mill y Morrison, 1992; Gartner, 1993; Walmsley y Jenkins, 1993; Um, 1993; Dann, 1996; Baloglu, 1997, 1999; Baloglu y McCleary, 1999a; Beerli Palacio y Martín Santana, 2004a y b). En particular, los estudios asocian la influencia de las motivaciones al componente afectivo de la imagen (Dann, 1996; Baloglu, 1997; Baloglu y McCleary, 1999a; Beerli Palacio y Martín Santana, 2004a y b); de forma similar, Gartner (1993) sugiere que el componente 
cognitivo de la imagen puede concebirse como la suma de creencias y evaluaciones del turista sobre los atributos del destino, mientras que el afectivo está vinculado con las motivaciones y los deseos del individuo en la elección del destino.

El objetivo principal de este trabajo consiste en identificar la naturaleza cognitivo-afectiva de la imagen del destino y estudiar si las motivaciones turísticas actúan como factores personales formadores de esta imagen. El objeto de estudio concreto-aplicado es la imagen percibida del turismo rural en Galicia; las razones que justifican su elección se relacionan con la significativa expansión de esta modalidad turística en la comunidad gallega, pues aunque representa un pequeño porcentaje de turistas adquiere un profundo significado social debido a su difusión por todo el territorio.

\section{El proceso de formación de la imagen turística: perspectiva de estudio desde los factores personales La multidimensionalidad de la imagen turística}

Un primer acercamiento a la delimitación teórica de la imagen turística exige reconocer la falta de una estructura conceptual sólida (Fakeye y Crompton, 1991; Echtner y Ritchie, 1991 y 1993; Gartner, 1993; Walmsley y Young, 1998; Beerli Palacio y Martín Santana, 2004b). Una definición que recoge las distintas dimensiones que de acuerdo con la literatura debe integrar este concepto es la propuesta por Baloglu y McCleary (1999b: 330), para quienes la imagen es la "representación mental de las creencias, los sentimientos y la impresión global del individuo sobre un destino turístico”. En concreto, incluye las evaluaciones tanto cognitivas (creencias o conocimientos que posee un individuo acerca de las características de un destino turístico) como afectivas (los sentimientos hacia el destino).

Los estudios más actuales (Baloglu y McCleary, 1999a; Yoon y Kim, 2000; Sönmez y Sirakaya, 2002; Kim y Richardson, 2003; Beerli Palacio y Martín Santana, 2004a y b; Pike y Ryan, 2004) evidencian que la imagen debe considerarse como un fenómeno multidimensional que se constituye mediante la incorporación de dos dimensiones interrelacionadas: a) las evaluaciones perceptuales/cognitivas, que se refieren a las creencias y los conocimientos que el turista posee sobre los atributos del destino (Baloglu, 1999; Pike y Ryan, 2004), y b) las evaluaciones afectivas, representadas por los sentimientos del turista hacia el destino (Chen y Uysal, 2002; Kim 
y Richardson, 2003). Asimismo, la combinación de estos dos elementos da lugar a una imagen global o compuesta que alude a la valoración positiva o negativa que se pueda tener del producto turístico (Leisen, 2001; Milman y Pizam, 1995).

\section{El efecto moderador de las motivaciones, las características sociodemográficas y los valores culturales sobre la imagen percibida del destino}

Merece señalarse el modelo de Baloglu y McCleary (1999a) como una completa aproximación al proceso de formación de la imagen turística. Estos autores desarrollan un paradigma que sistematiza los elementos principales que influyen en la imagen y los agrupan en dos categorías: factores personales y factores estímulo. Los primeros representan las características sociales y psicológicas del consumidor turístico; los segundos parten de un estímulo externo y se asocian con la tipología y la variedad de fuentes de información, la experiencia previa del individuo y los elementos de distribución.

Además, los factores personales hacen referencia a las motivaciones psicológicas del individuo (Moutinho, 1987; Stabler, 1990; Um, 1993; Baloglu, 1999), así como a las características sociodemográficas y los valores culturales del medio donde se desarrolla (Woodside y Lysonski, 1989; Um y Crompton, 1990; Stern y Krakover, 1993; Mackay y Fesenmaier, 1997; Baloglu, 2001).

\section{LAS MOTIVACIONES DEL TURISTA}

Las motivaciones, definidas como fuerzas sociopsicológicas que predisponen a los individuos a optar y participar en una actividad turística (Pizam, Neumann y Reichel, 1978; Raghep y Beard, 1982; Iso-Ahola, 1982), están incluidas en los modelos de elección de un destino y en los de formación de la imagen como factores de gran influencia (Stabler, 1990; Um y Crompton, 1990; Baloglu y McCleary, 1999a).

Se tiende a explicar las motivaciones que subyacen en el comportamiento turístico a través del marco conceptual push-pull, el cual recoge los factores de empuje y de atracción (Dann, 1977; Chon, 1989; Cha, McCleary y Uysal, 1995; Klenosky, 2002). Los primeros pueden describirse como fuerzas internas que predisponen al individuo a tomar la decisión de viajar (Dann, 1977, 1981; Baloglu y Uysal, 1996), 
es decir, están vinculados con los aspectos internos y emocionales del individuo (Yoon y Uysal, 2005). Por su parte, los factores de atracción representan las características del destino que persuaden a la persona (Kim y Lee, 2002; Klenosky, 2002) $\mathrm{y}$, a diferencia de las fuerzas de empuje, se relacionan con los aspectos externos y cognitivos (Yoon y Uysal, 2005).

Diversos estudios evidencian un significativo efecto de las motivaciones en la conformación de la imagen turística (Mayo y Jarvis, 1981; Stabler, 1990; Um y Crompton, 1990; Walmsley y Jenkins, 1993; Dann, 1996; Baloglu, 1997, 1999; Baloglu y McCleary, 1999a). En efecto, Mayo y Jarvis (1981: 30) indican que las motivaciones de los turistas condicionan las imágenes de los destinos, y Moutinho (1987: 18) asegura que, ya sea de manera consciente o inconsciente, participan en su configuración.

No obstante, algunos autores han planteado que las motivaciones sólo implican el componente afectivo de la imagen (Gartner, 1993; Dann, 1996; Baloglu, 1997), mientras que el componente cognitivo supone las creencias del individuo sobre un destino turístico; se sugiere un estrecho lazo entre las motivaciones psicológicas y la imagen afectiva (Dann, 1996; Baloglu, 1997).

Como señala Gartner (1993), el componente afectivo es el valor que las personas atribuyen a los destinos sobre la base de las motivaciones. En este sentido, las motivaciones influirán positivamente en la imagen turística cuando exista determinada correspondencia entre los beneficios buscados por el individuo y la naturaleza del destino (Beerli Palacio y Martín Santana, 2004a y b).

De este modo, la literatura respalda una importante influencia de los motivos turísticos sobre el componente afectivo de la imagen turística (Gartner, 1993; Walmsley y Jenkins, 1993; Dann, 1996; Baloglu, 1997; Baloglu y McCleary, 1999a; Beerli Palacio y Martín Santana, 2004a y b). Asimismo, Beerli Palacio y Martín Santana (2004a y b) sostienen que el impacto resulta positivo cuando hay correspondencia entre las motivaciones del turista y la naturaleza del destino. Más concretamente, estos autores apuntan que los individuos perciben de un modo más positivo el destino turístico en aquellos atributos que coinciden con sus motivaciones o beneficios buscados.

De acuerdo con estos argumentos, así como con la necesidad de profundizar en el estudio de los factores internos en la formación de la imagen de destino, se postula la siguiente hipótesis: los motivos turísticos del individuo tienen una influencia significativa en la dimensión afectiva de la imagen percibida del destino turístico. 


\section{LAS CARACTERÍSTICAS SOCIODEMOGRÁFICAS DEL TURISTA}

Prácticamente todos los modelos del proceso de decisión en la selección de los destinos turísticos afirman que las características sociodemográficas de los individuos, tales como sexo, edad, ocupación profesional, nivel de estudios, estado civil y clase social, entre otras, son variables internas que adquieren un protagonismo especial en la percepción de los destinos (Stabler, 1990; Stern y Krakover, 1993; Ahmed, 1994; Mackay y Fesenmaier, 1997; Baloglu y McCleary, 1999a; Beerli Palacio y Martín Santana, 2004a y b; Woodside y Lysonski, 1989; Um y Crompton, 1990).

Como mencionan Ashworth y Goodall (1990), la imagen turística varía entre los individuos, ya que en ella influye cómo éstos perciben los mensajes que se les comunican y, lógicamente, tienen sensibilidades distintas a la exposición de la información. Así pues, las características sociodemográficas se convierten en un auténtico filtro que condiciona la percepción de un destino turístico, la cual cambia de persona a persona (Beerli Palacio y Martín Santana, 2004a y b).

\section{LA PROCEDENCIA GEOGRÁFICO-CULTURAL DE LOS TURISTAS}

Los valores, costumbres y creencias de los individuos influyen notablemente no sólo en sus conductas en general, sino también en sus comportamientos de consumo y de ocio en particular (Richardson y Crompton, 1988).

La cultura, definida como el conjunto de creencias, valores, costumbres, ideas y normas de comportamiento acumulados por los seres humanos (Alonso y Grande, 2004), es por consiguiente un factor que puede filtrar la percepción del individuo y, en este sentido, Ahmed (1996) manifiesta que las diferencias regionales entre las personas implican la presencia de preferencias, sistemas de valores, normas, actitudes, estados mentales y subculturas muy distintos, lo cual puede estimular percepciones muy heterogéneas de un mismo destino turístico. En consecuencia, es natural pensar que grupos de individuos pertenecientes a distintos países 0 regiones, caracterizados, entonces, por poseer culturas diferenciadas, construyan imágenes diferentes sobre un mismo destino turístico (Richardson y Crompton, 1988; Chen y Kerstetter, 1999; Mackay y Fesenmaier, 2000; Hui y Wan, 2003; Beerli Palacio y Martín Santana, 2004a y b). 
La mayoría de los trabajos empíricos que han tratado de analizar las diferencias en la imagen percibida de los destinos turísticos en función de la distancia geográfico-cultural se ha centrado en el estudio de las zonas geográficas de procedencia de los turistas (Beerli Palacio y Martín Santana, 2004b).

\section{Metodología}

Para dar respuesta a los objetivos propuestos se plantea un proceso metodológico que combina una investigación inicial cualitativa y otra posterior cuantitativa. La fase cualitativa se apoya en la investigación exploratoria efectuada mediante una exhaustiva revisión bibliográfica y un grupo de discusión con los estudiantes del Máster Oficial en Dirección y Planificación del Turismo de la Universidad de La Coruña, cuya finalidad es conseguir la mayor cantidad de información sobre la problemática de estudio. Los resultados obtenidos en esta fase se toman como referencia para el diseño de la fase cuantitativa, en la cual se aplica una encuesta a los individuos que se encontraron realizando turismo rural en Galicia entre diciembre de 2008 y marzo de 2009, a partir de un cuestionario autoadministrado estructurado que se envía previamente por correo postal a los propietarios de los establecimientos de turismo rural gallegos contactados vía telefónica con anterioridad (véase cuadro 1).

CUADRO 1. Ficha técnica del proceso metodológico

\section{Proceso metodológico de la investigación}

Tipo de encuesta

Universo/población

Ámbito geográfico

Marco muestral

Tamaño muestral

\section{Encuesta autoadministrada por correo postal}

Turistas mayores de 16 años que realizaron turismo rural en Galicia y pernoctaron al menos una noche en un establecimiento de turismo rural

Comunidad Autónoma de Galicia

Turistas que realizaron turismo rural en Galicia y pernoctaron al menos una noche en un establecimiento de turismo rural ( $\mathrm{N}$ no determinado, se supone superior a 100000 unidades)

391 turistas rurales

(Continúa) 


\section{CUADRO 1. Ficha técnica del proceso metodológico}

(Finaliza)

\section{Proceso metodológico de la investigación}

\section{Tipo de encuesta}

Procedimiento de muestreo

Error muestral/nivel de confianza

\section{Encuesta autoadministrada por correo postal}

Muestreo estructural por cuotas de provincia y tipo de establecimiento

$+-5.5 \%$ para $\mathrm{p}=\mathrm{q}=0.5 \mathrm{yz}=2$

Colaboraron en la investigación un total de 154 casas rurales gallegas con la siguiente distribución: La Coruña: 47; Lugo: 36; Orense: 32; Pontevedra: 39. El volumen de cuestionarios útiles fue de 391 , que representan $19.53 \%$ de respuesta real, lo que permitió asumir un error muestral $+-5.5 \%$ para $p=q=0.5 \mathrm{y}$ $z=2$. En el cuadro 2 se presenta el perfil sociodemográfico de los turistas rurales encuestados.

CUADRO 2. Perfil sociodemográfico de la muestra

\begin{tabular}{lrrrrr}
\multicolumn{1}{c}{ Variables } & N & \multicolumn{1}{c}{ \% } & \multicolumn{1}{c}{ Variables } & N & \% \\
Sexo & & & Edad & 30 & 7.7 \\
Hombre & 184 & 47.1 & Entre 16 y 24 años & 127 & 32.5 \\
Mujer & 207 & 52.9 & Entre 25 y 34 años & 154 & 39.4 \\
& & & Entre 35 y 44 años & 56 & 14.3 \\
& & & Entre 45 y 54 años & 13 & 3.3 \\
& & & Entre 55 y 64 años & 0 & 0 \\
& & & 65 años 0 más & 11 & 2.8 \\
& & & NS/NC & & \\
Nivel de estudios & & & Ocupación & 83 & 21.2 \\
Sin estudios & - & - & Trabajador por cuenta propia & 256 & 65.5 \\
Estudios primarios & 17 & 4.3 & Trabajador por cuenta ajena & 11 & 2.8 \\
Estudios secundarios & 112 & 28.6 & Parado & 3 & 0.8 \\
Estudios universitarios & 261 & 66.8 & Jubilado/pensionista & 12 & 3.1 \\
NS/NC & 1 & 0.3 & Estudiante & & \\
\hline
\end{tabular}


CUADRo 2. Perfil sociodemográfico de la muestra

(Finaliza)

\begin{tabular}{|c|c|c|c|c|c|}
\hline Variables & $\mathbf{N}$ & $\%$ & Variables & $\mathbf{N}$ & $\%$ \\
\hline \multirow[t]{3}{*}{ Sexo } & & & Edad & & \\
\hline & & & Tareas del hogar & 20 & 5.1 \\
\hline & & & $\mathrm{NS} / \mathrm{NC}$ & 6 & 1.5 \\
\hline \multicolumn{3}{|l|}{ Estado civil } & \multicolumn{3}{|c|}{ Renta familiar mensual (estimada en 2000 euros) } \\
\hline Soltero & 135 & 34.5 & Por debajo de la media & 35 & 9.0 \\
\hline Casado & 185 & 47.3 & Similar a la media & 243 & 62.1 \\
\hline Viviendo en pareja & 60 & 15.3 & Por encima de la media & 98 & 25.1 \\
\hline Separado/divorciado & 6 & 1.5 & $\mathrm{NS} / \mathrm{NC}$ & 15 & 3.8 \\
\hline Viudo & 2 & 0.5 & $\begin{array}{l}\text { CCAA de procedencia* } \\
\text { (turistas rurales de origen español) }\end{array}$ & & \\
\hline $\mathrm{NS} / \mathrm{NC}$ & 3 & 0.8 & Andalucía & 9 & 2.3 \\
\hline \multicolumn{3}{|l|}{ Densidad del hábitat } & Asturias & 7 & 1.8 \\
\hline Rural & 87 & 22.2 & Cantabria & 15 & 3.9 \\
\hline Urbano & 301 & 77.0 & Castilla la Mancha y Castilla León & 5 & 1.3 \\
\hline \multirow[t]{4}{*}{$\mathrm{NS} / \mathrm{NC}$} & 3 & 0.8 & Cataluña & 19 & 4.9 \\
\hline & & & Galicia & 229 & 59.5 \\
\hline & & & Madrid & 84 & 21.8 \\
\hline & & & País Vasco & 13 & 3.4 \\
\hline
\end{tabular}

* Se refiere a los turistas rurales provenientes de España. En la muestra completa aparecen seis turistas rurales extranjeros: cuatro portugueses y dos franceses.

\section{Diseño de las escalas de medición de las variables}

A fin de medir el componente cognitivo, se han tenido en cuenta los atributos reflejados con más frecuencia en trabajos previos (Gartner, 1989; Fakeye y Crompton, 1991; Gartner y Shen, 1992; Echtner y Ritchie, 1993; Hu y Ritchie, 1993; Stabler, 1990; Baloglu y McCleary, 1999a y b; Chen y Hsu, 2000; Beerli Palacio y Martín Santana, 2004a y b); en concreto, se eligieron 20 atributos cognitivos sobre los que se recoge la opinión del turista mediante una escala Likert de siete posiciones $(1=$ total desacuerdo; 7 = total acuerdo).

Para la evaluación del componente afectivo se empleó una escala bipolar de diferencial semántico de cuatro ítems y siete puntos: aburrido-divertido, estresanterelajante, deprimente-excitante, desagradable-agradable, siguiendo la propuesta de 
Russel y Pratt (1980) utilizada con anterioridad (Walmsley y Young, 1998; Baloglu y McCleary, 1999a; Baloglu, 2001; Baloglu y Mangaloglu, 2001; Kim y Richardson, 2003; Beerli Palacio y Martín Santana, 2004a y b; Pike y Ryan, 2004).

Respecto a la medición de los motivos turísticos, se ha desarrollado una clasificación de éstos de acuerdo con las propuestas empleadas en investigaciones previas (Crompton, 1979; Dann, 1981; Iso-Ahola, 1982; Beard y Raghep, 1983; Fodness, 1994; Baloglu y McCleary, 1999a; McIntosh, Goeldner y Ritchie, 1999; Beerli Palacio y Martín Santana, 2004a y b). Así pues, en el cuestionario se ha incluido una lista de 19 motivos, el turista debe señalar los que lo han llevado a visitar el destino y el grado de importancia que concede a cada uno de ellos a través de una escala Likert $(1=$ poco importante; 7 = muy importante $)$.

\section{Análisis de resultados \\ Identificación de la naturaleza multidimensional de la imagen turística}

Con el objetivo de identificar las dimensiones de la imagen subyacentes al conjunto de atributos señalados, se realiza un análisis factorial exploratorio de componentes principales con rotación varimax. Sobre la base de los resultados expuestos en el cuadro 3 se evidencia una estructura compuesta por seis factores que explican más de $60 \%$ de la varianza total acumulada.

CUADRO 3. Análisis factorial exploratorio de la imagen

\section{Variables}

-Es un lugar con alojamientos rústicos de calidad

-En general, existe una buena relación calidad/precio

-Es un lugar sin contaminación ambiental, limpio y cuidado

-Los residentes de las zonas rurales de Galicia son amables y hospitalarios
Factores

$\begin{array}{llllll}1 & 2 & 3 & 4 & 5 & 6\end{array}$

0.795

0.782

0.780

0.777 
CUADRO 3. Análisis factorial exploratorio de la imagen

(Continuación)

\section{Variables}

-Es un lugar con buena atención al turista y que cuida la calidad del servicio

-Dispone de numerosas atracciones culturales para visitar (museos, monumentos históricos, etc.)

-Es un lugar con riqueza histórica y patrimonial

-Sus costumbres (artesanías, folclor, etc.) son dignas de conocer

-Presenta actividades culturales de mucho interés (festivales, celebraciones populares, etc.)

-Es un lugar que posee numerosos edificios y construcciones emblemáticas (iglesias, pazos, torres, etc.)

-Ofrece muchas oportunidades para la aventura y el deporte (senderismo, escalada, montañismo, etc.)

-Presenta un fácil acceso/transporte desde otras regiones

-Su clima resulta agradable

-Es un lugar donde existen zonas de recreo y disfrute para los niños

-Es un lugar sin demasiados turistas

-Es un lugar donde se facilita pasear con tranquilidad por el conjunto natural y/o histórico

-Es un lugar seguro para visitar

\section{Factores}

$\begin{array}{llllll}1 & 2 & 3 & 4 & 5 & 6\end{array}$

0.772

0.791

0.789

0.782

0.766

0.743
0.703

0.674

0.646

0.644

-Es un lugar que posee un ambiente relajado y tranquilo

-Dispone de gran belleza paisajística y áreas naturales muy hermosas

-Es un lugar donde puede degustarse con calidad gastronomía local rica y variada 
CUADRO 3. Análisis factorial exploratorio de la imagen

(Finaliza)

\section{Variables}

Factores

$\begin{array}{llllll}1 & 2 & 3 & 4 & 5 & 6\end{array}$

-El turismo rural en Galicia es un destino desagradable-

agradable

-El turismo rural en Galicia es un destino deprimente-

excitante

-El turismo rural en Galicia es un destino estresante-

relajante

-El turismo rural en Galicia es un destino aburrido-

divertido

\begin{tabular}{lrrrrrr} 
Número de ítems & \multicolumn{1}{c}{5} & \multicolumn{1}{c}{5} & \multicolumn{1}{c}{4} & \multicolumn{1}{c}{3} & \multicolumn{1}{c}{3} & \multicolumn{1}{c}{4} \\
Valor Eigen & 7.073 & 2.229 & 1.663 & 1.545 & 1.383 & 1.146 \\
\% Varianza explicada & 29.472 & 9.289 & 6.927 & 6.439 & 5.762 & 4.775 \\
\% Varianza acumulada & 29.472 & 38.761 & 45.688 & 52.127 & 57.789 & 62.664 \\
Alfa de Cronbach & 0.881 & 0.871 & 0.707 & 0.733 & 0.662 & 0.530 \\
\hline
\end{tabular}

Determinante Matriz Correlaciones $=3.58 \mathrm{E}-005$

Prueba de Esfericidad de Barlett $=3902.510(\mathrm{gl}=276 ; \mathrm{Sig}=0.000)$

Índice $\mathrm{KMO}=0.862$

Alfa de Cronbach (24 ítems) $=0.885$

F1: Oferta de alojamientos rústicos y entorno socioeconómico; F2: Atractivos histórico-culturales;

F3: Infraestructura general y facilidades turísticas y recreativas; F4: Atmósfera del destino;

F5: Ambiente social y atractivos naturales y gastronómicos; F6: Imagen afectiva.

La primera dimensión, con más peso en la estructura factorial examinada, está compuesta por cinco atributos y se llamó "Oferta de alojamientos rústicos y entorno socioeconómico”; el segundo factor reúne también cinco ítems y se denominó "Atractivos histórico-culturales"; el tercero, con cuatro ítems, "Infraestructura general y facilidades turísticas y recreativas"; el cuarto, "Atmósfera del destino", y el quinto, "Ambiente social y atractivos naturales y gastronómicos", ambos con tres ítems; y el sexto y último, "Imagen afectiva”, contiene los cuatro atributos relativos a los sentimientos y emociones que caracterizan el destino.

Se identifica, pues, una estructura bidimensional cognitivo-afectiva; en efecto, a la imagen del turismo rural en Galicia la integran, por un lado, cinco factores que hacen referencia a las percepciones cognitivas, y por otro, uno relacionado con las evaluaciones emocionales o afectivas. 


\section{Análisis del efecto moderador de las motivaciones en la formación de la imagen turística \\ ANÁLISIS DESCRIPTIVO DE LOS MOTIVOS TURÍSTICOS}

Debido al peso que tienen las motivaciones en el comportamiento y la elección del turista, es necesario conocer cuáles son las que más influyen en sus hábitos de viaje y en la selección del destino. En el cuadro 4 se detalla la distribución de los motivos que han señalado los sujetos de la muestra para visitar el destino y la importancia que otorgan a cada uno.

CUADRO 4. Análisis descriptivo de los motivos turísticos

Motivos

-Desconectar / cambiar de aires / salir de la rutina diaria

-Descansar / relajarme

-Aliviar el estrés y la tensión acumulados

-Disfrutar y estar en contacto directo con la naturaleza

-Disfrutar del tiempo libre con mi familia y/o amigos

-Buscar diversión. aventuras y/o emociones fuertes

-Ir a lugares pintorescos o con encanto

-Ir a lugares confortables con buenos alojamientos y/o restaurantes

-Disfrutar de la gastronomía típica de la zona

-Enriquecerme intelectual y/o espiritualmente

-Conocer sus paisajes y su entorno natural (montañas, ríos, parques, etc.)

-Conocer su patrimonio histórico-cultural (castillos, museos, etc.)

-Conocer mi entorno próximo
Motivos Grado de

existentes importancia

$\mathrm{N}(\%)$ de los motivos

Media (DT)

$377(96.4) \quad 6.01(1.020)$

$373(95.4) \quad 5.94(1.088)$

$342(87.5) \quad 5.75(1.150)$

$351(89.8) \quad 6.02(0.980)$

342 (87.5) $5.90(1.010)$

$241(61.6) \quad 4.64$ (1.410)

$330(84.4) \quad 5.82(1.093)$

$322(82.4) \quad 5.76(1.141)$

333 (85.2) $5.90(1.110)$

$266(68.1) \quad 5.09(1.419)$

335 (85.7) $5.84(1.306)$

$318(81.3) \quad 5.69(1.243)$

291 (74.4) $5.23(1.214)$ 
CUADRO 4. Análisis descriptivo de los motivos turísticos

(Finaliza)

Motivos

(Finaliza)

Motivos

-Conocer gente nueva

-Conocer su forma de vida e integrarme en las actividades de la gente local

-Encontrarme con personas de intereses similares

-Dar a conocer el lugar a amigos/familiares que no han estado

-Poder contar mis aventuras de viaje

-Ir a un destino que está de moda

\begin{tabular}{cc}
$\begin{array}{c}\text { Motivos } \\
\text { existentes } \\
\mathbf{N}(\%)\end{array}$ & $\begin{array}{c}\text { Grado de } \\
\text { importancia } \\
\text { de los } \\
\text { motivos } \\
\text { Media (DT) }\end{array}$ \\
\hline $239(61.1)$ & $4.05(1.481)$ \\
$244(62.4)$ & $4.06(1.471)$ \\
$248(63.4)$ & $4.01(1.548)$ \\
$239(61.1)$ & $3.95(1.975)$ \\
\hline $221(56.3)$ & $3.32(1.910)$ \\
$213(54.2)$ & $2.62(1.674)$
\end{tabular}

Nota: Los turistas indican la importancia que han concedido a cada una de las razones para realizar turismo rural en Galicia mediante una escala Likert ( 1 = muy poco importante; 7 = muy importante).

Aproximadamente $96 \%$ de los turistas declara que desconectar, cambiar de aires y/o salir de la rutina son sus principales motivos para acudir a Galicia a realizar turismo rural. También descansar y relajarse (95.4\%) son comúnmente admitidos; asimismo, casi $90 \%$ de los individuos se siente motivado por disfrutar y estar en contacto directo con la naturaleza, y argumenta que aliviar el estrés y la tensión acumulados, así como disfrutar del tiempo libre con la familia y/o amigos (ambos con $87.5 \%$ ), son razones de relevancia para realizar turismo rural en Galicia. Por otra parte, ir a un destino de moda, poder contar las aventuras de viaje, dar a conocer el lugar a amigos y/o familiares o conocer gente nueva muestran los porcentajes más bajos (entre 55 y $60 \%$ ).

En el cuadro 4 se puede advertir entonces que los motivos más valorados son disfrutar y estar en contacto directo con la naturaleza (6.02); desconectar, cambiar de aires y salir de la rutina (6.01), y descansar (5.94) son, a su vez, los más habituales que declaran los turistas para visitar el destino en cuestión. Entre aquellos menos significativos con base en la valoración de la muestra obtenida destacan ir a un destino que está de moda (2.62), poder contar las aventuras de viaje (3.32) y dar a conocer el lugar a amigos y familiares que no han estado ahí (3.95). 


\section{ANÁLISIS FACTORIAL EXPLORATORIO DE LOS MOTIVOS TURÍSTICOS}

Para identificar en qué dimensiones se agrupan los 19 motivos se lleva a cabo un análisis factorial exploratorio con el método de rotación varimax cuyos resultados finales aparecen en el cuadro 5. Así, fueron identificados cinco factores motivacionales con un notable porcentaje de varianza explicada (74.44\%).

El primer factor está integrado por cinco motivos referidos al entretenimiento en el destino turístico y se cataloga como "Diversión y entretenimiento"; para el segundo factor se considera apropiada la etiqueta "Conocimiento"; el tercero consta de cuatro motivos - al igual que el segundo- que guardan relación con la interacción social en el destino y se opta por calificarlo como "Socialización”; el cuarto, con tres motivos, recibe el nombre de "Descanso", y, finalmente, el quinto, también con tres motivos, se denomina "Prestigio".

CUADRO 5. Análisis factorial exploratorio de los motivos turísticos

\section{Variables}

-Ir a lugares confortables con buenos alojamientos y/o restaurantes

-Disfrutar de la gastronomía típica de la zona

-Disfrutar del tiempo libre con mi familia y/o amigos

-Ir a lugares pintorescos o con encanto

-Disfrutar y estar en contacto directo con la naturaleza

\section{Factores}

MOT1 MOT2 MOT3 MOT4 MOT5

0.770

0.766

0.759

0.719

-Conocer sus paisajes y su entorno natural (montañas, ríos, parques, etc.)

-Conocer mi entorno próximo

-Conocer su patrimonio histórico-cultural (castillos, museos, etc.) 
CUADRO 5. Análisis factorial exploratorio de los motivos turísticos

(Finaliza)

\begin{tabular}{|c|c|c|c|c|c|}
\hline \multirow{2}{*}{ Variables } & \multicolumn{5}{|c|}{ Factores } \\
\hline & MOT1 & MOT2 & MOT3 & MOT4 & MOT5 \\
\hline -Conocer gente nueva & & & 0.863 & & \\
\hline $\begin{array}{l}\text {-Conocer su forma de vida e integrarme en las } \\
\text { actividades de la gente local }\end{array}$ & & & 0.861 & & \\
\hline $\begin{array}{l}\text {-Buscar diversión, aventuras y/o emociones } \\
\text { fuertes }\end{array}$ & & & 0.443 & & \\
\hline -Descansar / relajarme & & & & 0.932 & \\
\hline $\begin{array}{l}\text {-Desconectar / cambiar de aires / salir de la } \\
\text { rutina diaria }\end{array}$ & & & & 0.887 & \\
\hline -Aliviar el estrés y la tensión acumulados & & & & 0.885 & \\
\hline -Poder contar mis aventuras de viaje & & & & & 0.937 \\
\hline $\begin{array}{l}\text {-Dar a conocer el lugar a amigos/familiares } \\
\text { que no han estado }\end{array}$ & & & & & 0.850 \\
\hline -Ir a un destino que está de moda & & & & & 0.813 \\
\hline Número de ítems & 5 & 4 & 4 & 3 & 3 \\
\hline Valor Eigen & 5.850 & 2.718 & 2.392 & 1.831 & 1.353 \\
\hline \% Varianza explicada & 30.791 & 14.305 & 12.587 & 9.635 & 7.122 \\
\hline$\%$ Varianza acumulada & 30.791 & 45.096 & 57.684 & 67.318 & 74.441 \\
\hline Alfa de Cronbach & 0.877 & 0.751 & 0.838 & 0.899 & 0.887 \\
\hline
\end{tabular}

Determinante Matriz Correlaciones $=1.29 \mathrm{E}-006$

Prueba de Esfericidad de Barlett $=1611.606(\mathrm{gl}=171 ; \mathrm{Sig}=0.000)$

Índice $\mathrm{KMO}=0.758$

Alfa de Cronbach (19 ítems) $=0.866$

MOT1: Diversión y entretenimiento; MOT2: Conocimiento; MOT3: Socialización; MOT4: Descanso; MOT5: Prestigio. 


\section{ESTIMACIÓN DE LA INFLUENCIA DE LOS MOTIVOS TURÍSTICOS}

\section{SOBRE LA IMAGEN DEL DESTINO}

Para estudiar la influencia que ejercen los motivos turísticos en la imagen afectiva del destino, se efectúa un análisis de regresión en el cual la imagen afectiva (F6) es la variable dependiente y las puntuaciones factoriales de las cinco dimensiones motivacionales identificadas previamente actúan como variables independientes (véase cuadro 6).

CUADRO 6. Influencia de los motivos turísticos sobre la imagen afectiva global*

\begin{tabular}{lccc}
\multicolumn{1}{c}{ Factores de los motivos } & $\begin{array}{c}\text { Coeficientes } \\
\text { Coeficientes Beta } \\
\text { estandarizados }\end{array}$ & Valor t & Sig. \\
MOT1: Diversión y entretenimiento & 0.175 & 0.815 & 0.417 \\
MOT2: Conocimiento & -0.026 & 2.103 & 0.038 \\
MOT3: Socialización & 0.184 & -3.312 & 0.756 \\
MOT4: Descanso & 0.311 & 2.211 & 0.029 \\
MOT5: Prestigio & 0.027 & 3.740 & 0.000 \\
\hline R Pearson: 0.403 & & 0.329 & 0.443 \\
R2 :0.163; R2 corregida: 0.128 & & & \\
Error típico de estimación: 0.922 & & & \\
Durbin Watson: 1.477 & & & \\
F: 4.701 (Signif. F: 0.001 ) & & & \\
\hline \hline
\end{tabular}

*Regresión lineal basada en las puntuaciones factoriales.

Los resultados evidencian que el coeficiente de correlación de Pearson tiene un valor de 0.403 , lo cual sugiere que existe una aceptable, aunque de poca consideración, asociación lineal entre las variables. El coeficiente de determinación R cuadrado indica que la proporción de la varianza de la imagen afectiva explicada por los factores motivacionales es de $16.3 \%$, un porcentaje bastante mediocre que apunta que tal vez los motivos turísticos no funcionen como buenos predictores de la imagen afectiva del destino. Además, con base en el valor de $\mathrm{F}=4.70$ y $p=0.001$ se deriva que el modelo presenta escasa predicción de la variable dependiente. 
De acuerdo con estas premisas, se desprende que mediante la observación del valor $t$ y su nivel de significación asociado, los únicos motivos que tienen una relación estadísticamente significativa con la dimensión afectiva de la imagen se centran, en orden decreciente de significatividad, en los siguientes factores: MOT4 “Descanso" ( $t=3.74 ; p=0.000)$, MOT3 “Socialización” ( $t=2.21 ; p=0.029)$ y MOT1 "Diversión y entretenimiento" $(t=2.10 ; p=0.038)$.

Los otros dos factores motivacionales, MOT2 "Conocimiento" y MOT5 "Prestigio”, no manifiestan relación significativa con la imagen afectiva. Esto podría deberse a que los motivos relacionados con conocer el paisaje y el entorno natural, el entorno próximo o el patrimonio histórico y cultural, así como el enriquecimiento intelectual y espiritual, por un lado, y, por otro, con poder contar las aventuras de viaje, dar a conocer el lugar a amigos y/o familiares que no han estado e ir a un destino que está de moda, son los menos relevantes para los turistas que visitan Galicia como destino de turismo rural.

\section{MODELOS ANOVA DEL IMPACTO DE LOS MOTIVOS SOBRE LA IMAGEN DEL DESTINO}

A continuación, se realizan varios modelos ANOVA, uno por cada factor motivacional que tiene relación estadísticamente significativa, con base en el análisis de regresión lineal previamente llevado a cabo sobre la imagen. Se consideran variables dependientes la imagen afectiva global - promedio de los ítems que la integran - y cada uno de los atributos afectivos de la imagen turística. Por su parte, la variable independiente está distribuida en tres categorías: 1) turistas que conceden poca importancia al factor motivacional en cuestión; 2) turistas que le conceden importancia media, y 3) turistas que le conceden elevada importancia.

El cuadro 7 contiene los resultados del ANOVA para el factor MOT1 "Diversión y entretenimiento". Se tuvo en cuenta el uso de la mediana (5.89) como punto de división para segmentar a estos viajeros en turistas que conceden poca importancia a los motivos de diversión y entretenimiento $(\mathrm{N}=22)$; los que le conceden importancia media $(\mathrm{N}=188)$, y quienes le conceden importancia elevada $(\mathrm{N}=93)$. 
CUADRO 7. Resultados del ANOVA según los motivos de diversión y entretenimiento

\begin{tabular}{|c|c|c|c|c|c|}
\hline \multirow{5}{*}{$\begin{array}{l}\text { Motivos de } \\
\text { diversión y } \\
\text { entretenimiento }\end{array}$} & \multirow{2}{*}{$\begin{array}{c}\text { Imagen } \\
\text { afectiva global }\end{array}$} & \multicolumn{4}{|c|}{ Atributos de la imagen afectiva } \\
\hline & & $\begin{array}{l}\text { Aburrido- } \\
\text { divertido }\end{array}$ & $\begin{array}{l}\text { Estresante- } \\
\text { relajante }\end{array}$ & $\begin{array}{l}\text { Deprimente- } \\
\text { excitante }\end{array}$ & $\begin{array}{l}\text { Desagradable- } \\
\text { agradable }\end{array}$ \\
\hline & Sig. & F Sig. & F $\quad$ Sig. & F Sig. & F Sig. \\
\hline & $\begin{array}{ll}7.007 & 0.001\end{array}$ & $3.548 \quad 0.030$ & $0.993 \quad 0.372$ & $\begin{array}{ll}7.736 & 0.001\end{array}$ & $5.747 \quad 0.004$ \\
\hline & $\mathrm{Eta}=0.211$ & Eta $=0.152$ & Eta $=0.081$ & $\mathrm{Eta}=0.221$ & Eta $=0.192$ \\
\hline $\begin{array}{l}\text { Grupo 1: } \\
\text { Turistas que } \\
\text { conceden poca } \\
\text { importancia } \\
\text { a los motivos } \\
\text { de diversión } \\
(\mathrm{N}=22)\end{array}$ & 6.05 & 5.91 & 6.82 & 5.05 & 6.41 \\
\hline $\begin{array}{l}\text { Grupo 2: } \\
\text { Turistas que } \\
\text { conceden } \\
\text { importancia } \\
\text { media a los } \\
\text { motivos de } \\
\text { diversión } \\
(\mathrm{N}=188)\end{array}$ & 6.15 & 5.83 & 6.69 & 5.56 & 6.61 \\
\hline $\begin{array}{l}\text { Grupo 3: } \\
\text { Turistas que } \\
\text { conceden } \\
\text { importancia } \\
\text { elevada a los } \\
\text { motivos de } \\
\text { diversión } \\
(\mathrm{N}=93)\end{array}$ & 6.31 & 5.95 & 6.74 & 5.84 & 6.70 \\
\hline
\end{tabular}

Del cuadro 7 se desprende que el factor "Diversión y entretenimiento" tiene una influencia significativa $(\mathrm{F}=7.007$; Sig. $=0.001)$ en la imagen afectiva global. El grupo tres es el que otorga una valoración media más elevada a la dimensión afectiva de la imagen (6.31). Cabe destacar que este factor tiene asimismo influencia sobre los atributos deprimente-excitante $(\mathrm{F}=7.736$; $\mathrm{Sig} .=0.001) \mathrm{y}$ desagradableagradable $(\mathrm{F}=5.747$; Sig. $=0.004)$. En ambos casos, el grupo tres es el que confiere una valoración más positiva. 
En el cuadro 8 se recoge la información del modelo ANOVA para el factor MOT3 "Socialización". Los turistas encuestados que alegaron el factor "Socialización" para acudir al destino fueron divididos según la mediana (4.25) en turistas que conceden poca importancia a los motivos de socialización $(\mathrm{N}=58)$; que le conceden importancia media $(\mathrm{N}=56)$, y los que le conceden importancia elevada $(\mathrm{N}=63)$.

\section{CUADRO 8. Resultados del ANOVA según los motivos de socialización}

\begin{tabular}{|c|c|c|c|c|c|}
\hline \multirow{5}{*}{$\begin{array}{l}\text { Motivos de } \\
\text { socialización }\end{array}$} & \multirow{2}{*}{$\begin{array}{l}\text { Imagen } \\
\text { afectiva global }\end{array}$} & \multicolumn{4}{|c|}{ Atributos de la imagen afectiva } \\
\hline & & $\begin{array}{l}\text { Aburrido- } \\
\text { divertido }\end{array}$ & $\begin{array}{l}\text { Estresante- } \\
\text { relajante }\end{array}$ & $\begin{array}{l}\text { Deprimente- } \\
\text { excitante }\end{array}$ & $\begin{array}{l}\text { Desagradable- } \\
\text { agradable }\end{array}$ \\
\hline & F $\quad$ Sig. & F $\quad$ Sig. & F $\quad$ Sig. & F Sig. & F $\quad$ Sig. \\
\hline & $\begin{array}{ll}7.315 \quad 0.001 & \end{array}$ & $1.685 \quad 1.188$ & $0.556 \quad 0.574$ & $7.636 \quad 0.001$ & $5.804 \quad 0.004$ \\
\hline & $\mathrm{Eta}=0.279$ & Eta $=0.138$ & $\mathrm{Eta}=0.080$ & $\mathrm{Eta}=0.284$ & $\mathrm{Eta}=0.250$ \\
\hline $\begin{array}{l}\text { Grupo 1: } \\
\text { Turistas que } \\
\text { conceden } \\
\text { poca } \\
\text { importancia a } \\
\text { los motivos de } \\
\text { socialización } \\
(\mathrm{N}=58)\end{array}$ & 6.08 & 5.86 & 6.79 & 5.28 & 6.40 \\
\hline $\begin{array}{l}\text { Grupo 2: } \\
\text { Turistas que } \\
\text { conceden } \\
\text { importancia } \\
\text { media a los } \\
\text { motivos de } \\
\text { socialización } \\
(\mathrm{N}=56)\end{array}$ & 6.21 & 5.93 & 6.73 & 5.54 & 6.63 \\
\hline $\begin{array}{l}\text { Grupo 3: } \\
\text { Turistas que } \\
\text { conceden } \\
\text { importancia } \\
\text { elevada a los } \\
\text { motivos de } \\
\text { socialización } \\
(\mathrm{N}=63 \text { ) }\end{array}$ & 6.35 & 5.95 & 6.81 & 5.94 & 6.68 \\
\hline
\end{tabular}


En los resultados expuestos en el cuadro 8 puede observarse que el factor "Socialización" posee una influencia estadísticamente significativa $(F=7.315$; Sig. $=0.001)$ en la imagen afectiva del destino. Asimismo, el grupo tres presenta una valoración más positiva de la imagen afectiva (6.35). Otro aspecto interesante es que este factor influye también sobre los atributos deprimente-excitante $(F=7.636$; Sig. $=0.001)$ y desagradable-agradable $(F=5.804$; Sig. $=0.004)$. En ambos casos, vuelve a ser el grupo tres el que valora estos dos atributos más positivamente.

El modelo ANOVA realizado sobre el factor MOT4 "Descanso" se ilustra en el cuadro 9. De igual manera que en los factores previos, los turistas que efectuaron el viaje por el motivo de "Descanso" fueron distribuidos en tres categorías de acuerdo con la mediana (5.92) en turistas que conceden poca importancia a los motivos de descanso $(\mathrm{N}=133)$; que le conceden importancia media $(\mathrm{N}=92)$, y quienes le conceden importancia elevada $(\mathrm{N}=113)$.

CUADRO 9. Resultados del ANOVA según los motivos de descanso

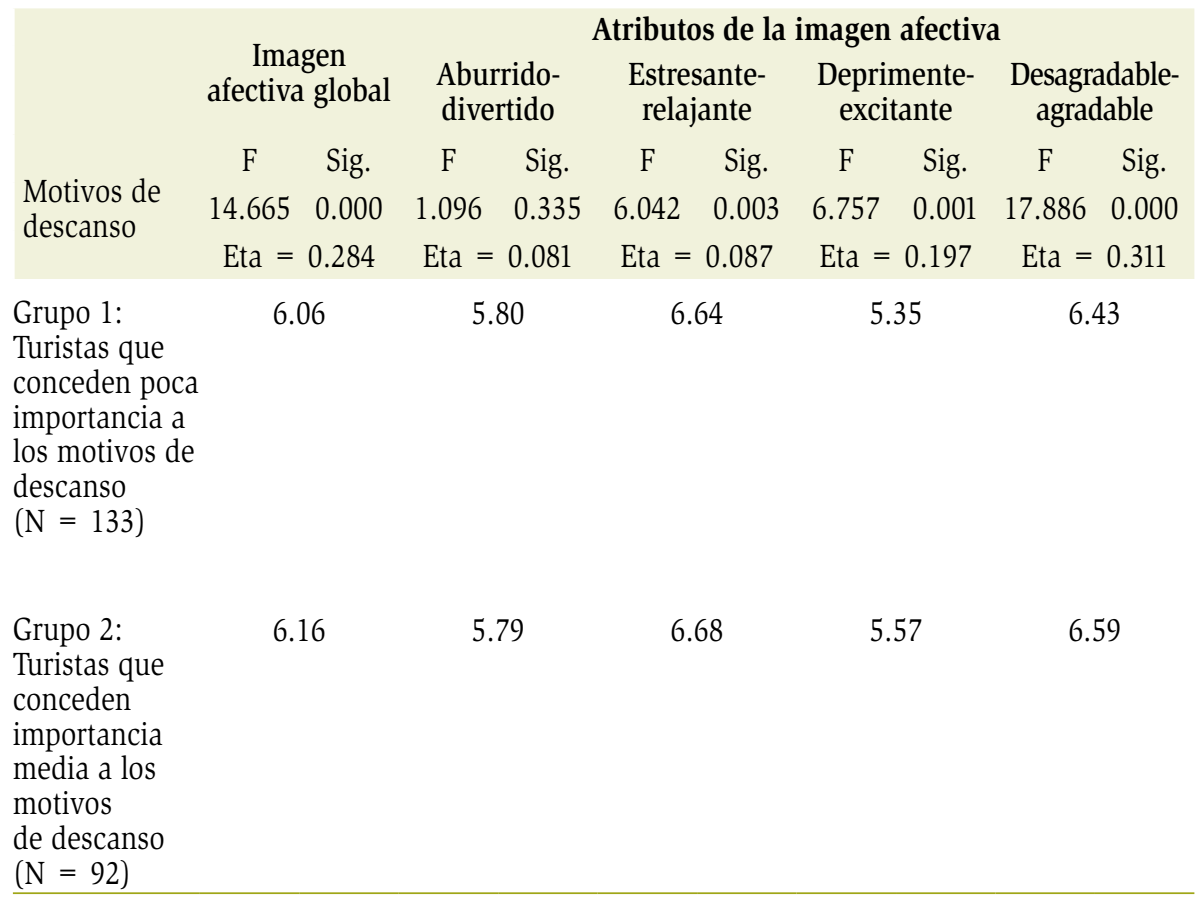


CUADRO 9. Resultados del ANOVA según los motivos de descanso

(Finaliza)

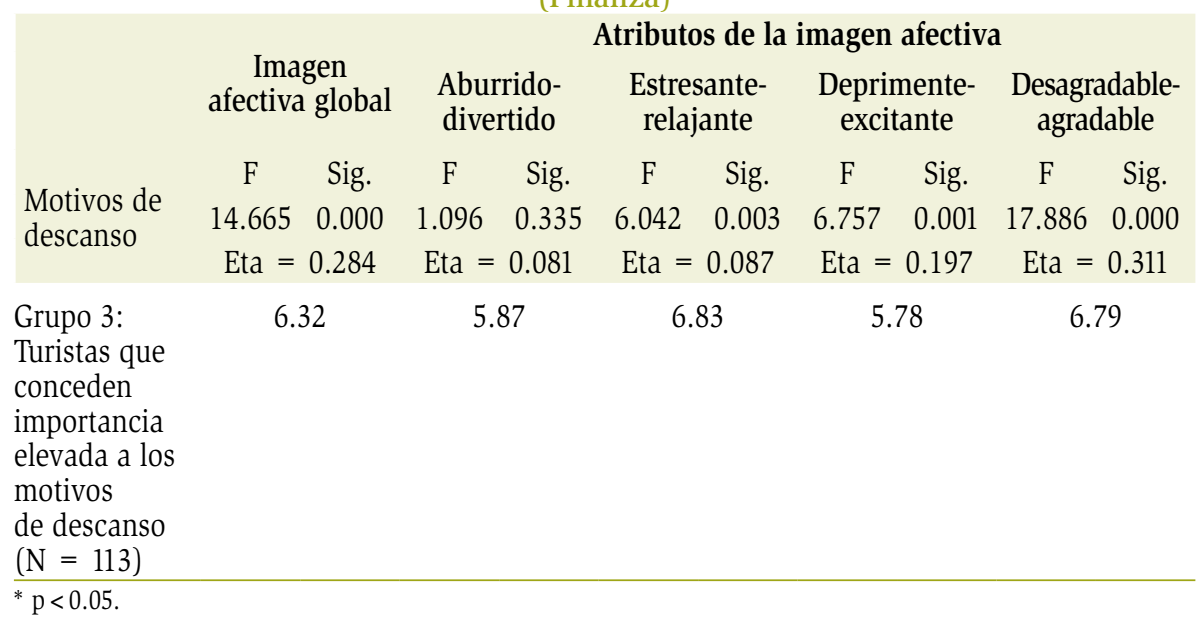

Se observa también que el factor "Descanso" posee un efecto estadísticamente significativo $(F=14.665$; Sig. $=0.000)$ sobre la imagen afectiva global. Este factor tiene además efectos sobre los atributos estresante-relajante $(F=6.042$; Sig. $=0.003)$, deprimente-excitante $(\mathrm{F}=6.757$; Sig. $=0.001)$ y desagradableagradable $(\mathrm{F}=17.886$; $\mathrm{Sig} .=0.000)$. En todos los casos en que este factor motivacional repercute sobre la imagen afectiva, es el tercer grupo el que manifiesta una valoración más positiva.

En síntesis, se demuestra empíricamente la influencia moderada de los motivos turísticos en la imagen afectiva del destino, puesto que de los cinco factores motivacionales sólo tres se relacionan de manera significativa con la imagen afectiva global: MOT1 "Diversión y entretenimiento", MOT3 “Socialización” y MOT4 "Descanso". Del mismo modo, estos tres factores motivacionales presentan una importancia significativa, aunque mediocre, sobre los atributos excitante-relajante y desagradable-agradable. No obstante, el factor MOT4 "Descanso" está vinculado también con el atributo estresante-relajante.

Estos resultados confirman, en una medida moderada, la siguiente hipótesis: los motivos del turista tienen una influencia significativa en la dimensión afectiva de la imagen percibida del destino. Por otra parte, se demuestra que cuanto más estrecha es la relación entre las motivaciones del turista y los atributos emocionales del destino, más positiva es la imagen afectiva de éste. 


\section{Conclusiones}

La mayoría de los recursos turísticos son de carácter objetivo y tangible; sin embargo, su imagen percibida puede ser diversa dada su naturaleza subjetiva (Bigné, Sánchez y Sánchez, 2001; Leisen, 2001; Gallarza, Gil y Calderón, 2002), lo que analíticamente implica situar en primer plano no los objetos/servicios turísticos disponibles, sino la manera en que los turistas los perciben.

La naturaleza de la imagen turística, los factores que participan en su formación y sus implicaciones en el comportamiento del turista son aspectos de enorme interés pero que requieren todavía un mayor esfuerzo investigador.

Los resultados de la medición de la imagen del turismo rural en Galicia revelan una estructura bidimensional integrada por un conjunto de cinco factores que hacen referencia a las percepciones cognitivas y un factor relacionado con las evaluaciones afectivas. En consecuencia, los individuos utilizan las distintas dimensiones de la imagen para comparar los destinos evocados en su mente una vez surgida la motivación de viajar y seleccionar, en última instancia, el destino turístico a visitar. Por tanto, para promocionar de manera eficaz un destino turístico no deben resaltarse exclusivamente sus recursos naturales, culturales o humanos, sino también las emociones o los sentimientos que puede provocar.

De los resultados se deriva también que la imagen es muy positiva en particular para los factores 5 "Ambiente social y atractivos naturales y gastronómicos" y 6 "Imagen afectiva", lo cual sugiere la necesidad de estudiar con mayor profundidad el componente afectivo de la imagen debido a su gran peso en la experiencia turística.

Así, en la promoción turística deben considerarse los factores personales, puesto que se demuestra empíricamente que éstos participan en la formación de la imagen. El heterogéneo procesamiento de la información de individuos con diferente perfil sociodemográfico y/o cultural, junto con la percepción más positiva de aquellos atributos afectivos que coinciden con sus motivos o beneficios buscados en el viaje, justifica segmentar el mercado con la intención de realizar una comunicación donde no se enfaticen todas las emociones que es capaz de producir el destino, sino sólo aquellas relacionadas con las principales motivaciones de cada grupo de turistas (San Martín Gutiérrez y Rodríguez del Bosque, 2010).

No obstante, la generalización de los resultados debe tomarse con cautela, pues la muestra utilizada posee características homogéneas respecto a los motivos turísticos 
existentes. Cabe agregar que la motivación es por lo general aceptada como uno de los conceptos centrales en la comprensión del comportamiento turístico y en el proceso de elección del destino; sin embargo, su identificación presenta ciertas dificultades, ya que el proceso de compra no se desencadena como consecuencia de una motivación única, sino que intervienen diferentes motivaciones interrelacionadas que, además, varían según el tiempo y el individuo (Valls, 2003).

Por otra parte, sólo se permite generalizar los datos obtenidos para la población de la muestra y el lugar en cuestión, ya que las características particulares del destino, así como el periodo en que se recoge la información, pueden condicionarlos; empero, este destino puede devenir representativo de otras zonas de turismo rural. 


\section{FUENTES CONSULTADAS}

Ahmed, Z. U. (1991). “The Influence of the Components of a State's Tourist Image on Product Positioning Strategy". Tourism Management, 12 (4), 331-340.

_ (1994). "Determinants of the Components of a State's Tourist Image and their Marketing Implications”. Journal of Hospitality \& Leisure Marketing, 2 (1), 55-69.

_ (1996). "The Need for the Identification of the Constituents of a Destination's Tourism Image: a Promotion Segmentation Perspective”. Journal of Professional Services Marketing, 14 (1), 37-60.

Alonso, J. e I. Grande (2004). Comportamiento del consumidor. Decisiones y estrategias de marketing. $5^{\text {a }}$ ed. Madrid: ESIC.

Ashworth, G. y B. Goodall (1990). “Tourist Images: Marketing Considerations”, en B. Goodall y G. Ashworth (eds.). Marketing in the Tourism Industry: The Promotion of Destination Regions. Londres: Routledge, 213-238.

Ashworth, G. y H. Voogd (1990). Selling the City: Marketing Approaches in Public Sector Urban Planning. Chichester: Wiley.

Baloglu, S. (1997). "The Relationship Between Destination Images and Sociodemographic and Trip Characteristics of International Travellers". Journal of Vacation Marketing, 3 (3), 221-233.

(1999). "A Path Analytic Model of Visitation Intention Involving Information Sources, Socio-Psychological Motivations, and Destination Image”. Journal of Travel \& Tourism Marketing, 8 (3), 81-91.

- (2001). "Image Variations of Turkey by Familiarity Index: Informational and Experiential Dimensions”. Tourism Management, 22, 27-133.

Baloglu, S. y K. W. McCleary (1999a). "A Model of Destination Image Formation". Annals of Tourism Research, 26 (4), 868-897.

(1999b). "Un modelo para la formación de la imagen de un destino". Annals of Tourism Research en Español, 1 (2), 325-355.

Baloglu, S. y M. Mangaloglu (2001). "Tourism Destinations Images of Turkey, Egypt, Greece, and Italy as Perceived by US-based tour operators and travel agents”. Tourism Management, 22, 1-9.

Baloglu, S. y M. Uysal (1996). "Market Segments of Push and Pull Motivations: a Canonical Correlation Approach”. International Journal of Contemporary Hospitality Management, 8 (3), 32-38. 
Beard, J. G. y M. G. Raghep (1983). “Measuring Leisure Motivation”. Journal of Leisure Research, 15, 219-228.

Beerli Palacio, A. y J. Martín Santana (2004a). "Factors Influencing Destination Image”. Annals of Tourism Research, 31 (3), 657-681.

- (2004b). "Tourist's Characteristic and the Perceived Image of Tourist Destinations: A Quantitative Analysis - A Case Study of Lanzarote, Spain”. Tourism Management, 25 (5), 623-636.

Bigné, J. E., M. I. Sánchez y J. Sánchez (2001). “Tourism Image, Evaluation Variables and after Purchase Behaviour: Inter-Relationship”. Tourism Management, 22, 607-616.

Bramwell, B. y L. Rawding (1996). “Tourism Marketing Images of Industrial Cities”. Annals of Tourism Research, 23 (1), 201-221.

Cha, S., K. W. McCleary y M. Uysal (1995). "Travel Motivations of Japanese Overseas Travellers: A Factor-cluster Segmentation Approach”. Journal of Travel Research, 34 (1), 33-39.

Chen, J. S. (2001). “A Case Study of Korean Outbound Travellers' Destination Images by using Correspondence Analysis”. Tourism Management, 22, 345-350.

Chen, J. S. y C. H. C. Hsu (2000). "Measurement of Korean Tourists' Perceived Images of Overseas Destinations”. Journal of Travel Research, 38 (4), 411-416.

Chen, J. S. y M. Uysal (2002). "Market Positioning Analysis: a Hybrid Approach”. Annals of Tourism Research, 29 (4), 987-1003.

Chen, P. J. y D. L. Kerstetter (1999). “International Students' Image of Rural Pennsylvania as a Travel Destination”. Journal of Travel Research, 37 (3), 256-266.

Chon, K-S. (1989). “Understanding Recreational Traveller's Motivation, Attitude and Satisfaction”. The Tourist Review, 44 (1), 3-7.

Crompton, J. L. (1979). "Motivation for Pleasure Vacation”. Annals of Tourism Research, 6 (4), 409-424.

Dann, G. M. S. (1977). “Anomie, Ego-Enhancement and Tourism”. Annals of Tourism Research, 4 (4), 184-194.

- (1981). "Tourist Motivation: an Appraisal”. Annals of Tourism Research, 8 (2), 187-219.

(1996). “Tourists' Images of a Destination - an Alternative Analysis”. Journal of Travel \& Tourism Marketing, 5 (1-2), 41-55. 
Echtner, C. M. y J. R. B. Ritchie (1991). "The Meaning and Measurement of Destination Image”. The Journal of Tourism Studies, 2 (2), 2-12.

(1993). "The Measurement of Destination Image: an Empirical Assessment". Journal of Travel Research, 31 (4), 3-13.

Fakeye, P. C. y J. L. Crompton (1991). "Image Differences Between Prospective, FirstTime and Repeat Visitors to the Lower Rio Grande Valley”. Journal of Travel Research, 30 (2), 10-16.

Fodness, D. (1994). "Measuring Tourist Motivation”. Annals of Tourism Research, 21 (3), 555-581.

Gallarza, M. G., I. Gil y H. Calderón (2002). "Imagen de un destino: Hacia un marco conceptual”. Annals of Tourism Research en Español, 4 (1), 37-62.

Gartner, W. C. (1989). “Tourism Image: Attribute Measurement of State Tourism Products using Multidimensional Scaling Techniques”. Journal of Travel Research, 28 (2), 16-20.

- (1993). "Image Formation Process". Journal of Travel \& Tourism Marketing, 2 (2-3), 191-215.

Gartner, W. C. y J. Shen (1992). “The Impact of Tiananmen Square on China's Tourism Image”. Journal of Travel Research, 30 (4), 47-52.

Hu, Y. y J. R. B. Ritchie (1993). "Measuring Destination Attractiveness: A Contextual Approach”. Journal of Travel Research, 32 (2), 25-34.

Hui, T. K. y T. W. D. Wan (2003). "Singapore's Image as a Tourist Destination”. International Journal of Tourism Research, 5 (4), 305-313.

Iso-Ahola, S. E. (1982). “Toward a Social Psychological Theory of Tourism Motivation: A Rejoinder”. Annals of Tourism Research, 9 (2), 256-262.

Kim, H. B. y S. L. Richardson (2003). "Motion Picture Impacts on Destination Images". Annals of Tourism Research, 30 (1), 216-237.

Kim, S-S. y C-K. Lee (2002). "Push and Pull Relationships”. Annals of Tourism Research, 29 (1), 257-260.

Klenosky, D. B. (2002). "The Pull of Tourism Destinations: A Means-end Investigation”. Journal of Travel Research, 40 (4), 385-395.

Leisen, B. (2001). “Image Segmentation: the Case of a Tourism Destination”. Journal of Services Marketing, 15 (1), 49-66.

Mackay, K. J. y D. R. Fesenmaier (1997). "Pictorial Element of Destination in Image Formation”. Annals of Tourism Research, 24 (3), 537-565. 
_ (2000). “An Exploration of Cross-cultural Destination Image Assessment”. Journal of Travel Research, 38 (4), 417-423.

Mayo, E. J. y L. P. Jarvis (1981). The Psychology of Leisure Travel. Boston: CBI Publishing Company.

McIntosh, R. W., C. R. Goeldner y J. R. Ritchie (1999). Turismo, planeación, administración y perspectivas. $2^{\text {a }}$ ed. México: Limusa Wiley.

Mill, R. C. y A. M. Morrison (1992). The Tourism System: An Introductory Text. $2^{\text {a }}$ ed. Englewood Cliffs: Prentice-Hall.

Milman, A. y A. Pizam (1995). "The Role of Awareness and Familiarity with a Destination: The Central Florida Case”. Journal of Travel Research, 33 (3), 21-27.

Moutinho, L. (1987). “Consumer Behavior in Tourism”. European Journal of Marketing, 21 (10), 5-44.

Pike, S. y C. Ryan (2004). "Destination Positioning Analysis Through a Comparison of Cognitive, Affective, and Conative Perceptions". Journal of Travel Research, 42 (4), 333-342.

Pizam, A., Y. Neumann y A. Reichel (1978). "Dimensions of Tourist Satisfaction with a Destination Area”. Annals of Tourism Research, 5 (3), 314-322.

Raghep, M. G. y J. G. Beard (1982). "Measuring Leisure Attitudes”. Journal of Leisure Research, 14 (2), 155-162.

Richardson, S. y J. L. Crompton (1988). "Cultural Variations in Perceptions of Vacation Attributes”. Tourism Management, 9 (2), 128-136.

Russel, J. A. y G. Pratt (1980). "A Description of the Affective Quality Attributed to Environments". Journal of Personality and Social Psychology, 38 (2), 311-322.

San Martín Gutiérrez, H. e I. Rodríguez del Bosque (2010). "Los factores estímulo y personales como determinantes de la formación de la imagen de marca de los destinos turísticos: un estudio aplicado a los turistas que visitan un destino vacacional”. Cuadernos de Economía y Dirección de la Empresa, 43, 37-63.

Sönmez, S. y E. Sirakaya (2002). "A Distorted Destination Image? The Case of Turkey". Journal of Travel Research, 41 (2), 185-196.

Stabler, M. J. (1990). "The Image of Destination Regions: Theoretical and Empirical Aspects”, en B. Goodall y G. Ashworth (eds.). Marketing in the Tourism Industry: The Promotion of Destination Regions. Londres: Routledge, 133-161.

Stern, E. y S. Krakover (1993). "The Formation of a Composite Urban Image”. Geographical Analysis, 25 (2), 130-146. 
Um, S. (1993). "Pleasure Travel Destination Choice", en M. Khan, M. Olsen y T. Var (eds.). VNR's Encyclopedia of Hospitality and Tourism. Nueva York: Wiley, 811-821.

Um, S. y J. L. Crompton (1990). "Attitude Determinants in Tourism Destination Choice”. Annals of Tourism Research, 17 (3), 432-448.

Valls, J. F. (2003). Las claves del mercado turístico. Cómo competir en el nuevo entorno. Bilbao: Ediciones Deusto.

Walmsley, D. J. y J. M. Jenkins (1993). “Appraisive Images of Tourist Areas: Application of Personal Construct”. Australian Geographer, 24 (2), 1-13.

Walmsley, D. J. y M. Young (1998). "Evaluative Images and Tourism: The Use of Personal Constructs to Describe the Structure of Destinations Images”. Journal of Travel Research, 36 (3), 65-69.

Woodside, A. G. y S. Lysonski (1989). "A General Model of Traveller Destination Choice”. Journal of Travel Research, 27 (4), 8-14.

Yoon, S-J. y J-H. Kim (2000). "An Empirical Validation of a Loyalty Model based on Expectation Disconfirmation”. Journal of Consumer Marketing, 17 (2), 120-136.

Yoon, Y. y M. Uysal (2005). "An Examination of the Effects of Motivation and Satisfaction on Destination Loyalty: a Structural Model”. Tourism Management, 26, 45-56. 